\title{
Contextual and Granular Policy Enforcement in Database-backed Applications
}

\author{
Abhishek Bichhawat \\ Carnegie Mellon University \\ Pittsburgh, PA, USA \\ Jean Yang \\ Carnegie Mellon University \\ Pittsburgh, PA, USA
}

\author{
Matt Fredrikson \\ Carnegie Mellon University \\ Pittsburgh, PA, USA \\ Akash Trehan* \\ Microsoft Vancouver \\ Vancouver, British Columbia, Canada
}

\section{KEYWORDS}

Database-backed applications; granular access policies; contextual access control; API-specific policies

Dabase-backed applications rely on inlined policy checks to pro cess users' private and confidential data in a policy-compliant manner as traditional database access control mechanisms cannot enforce complex policies. However, application bugs due to missed checks are common in such applications, which result in data breaches. While separating policy from code is a natural solution, many data protection policies specify restrictions based on the context in which data is accessed and how the data is used. Enforcing these restrictions automatically presents significant challenges, as the information needed to determine context requires a tight coupling between policy enforcement and an application's implementation.

We present Estrela, a framework for enforcing contextual and granular data access policies. Working from the observation that API endpoints can be associated with salient contextual information in most database-backed applications, Estrela allows developers to specify API-specific restrictions on data access and use. Estrela provides a clean separation between policy specification and the application's implementation, which facilitates easier auditing and maintenance of policies. Policies in Estrela consist of pre-evaluation and post-evaluation conditions, which provide the means to modulate database access before a query is issued, and to impose finergrained constraints on information release after the evaluation of query, respectively. We build a prototype of Estrela and apply it to retrofit several real world applications (from 1000-80k LOC) to enforce different contextual policies. Our evaluation shows that Estrela can enforce policies with minimal overheads.

\section{CCS CONCEPTS}

- Security and privacy $\rightarrow$ Access control; Software security engineering; Web application security.

\footnotetext{
${ }^{*}$ Work done while interning at Carnegie Mellon University.

Permission to make digital or hard copies of all or part of this work for personal or classroom use is granted without fee provided that copies are not made or distributed for profit or commercial advantage and that copies bear this notice and the full citation on the first page. Copyrights for components of this work owned by others than the author(s) must be honored. Abstracting with credit is permitted. To copy otherwise, or republish, to post on servers or to redistribute to lists, requires prior specific permission and/or a fee. Request permissions from permissions@acm.org.

ASIA CCS '20, October 5-9, 2020, Taipei, Taiwan

(C) 2020 Copyright held by the owner/author(s). Publication rights licensed to ACM. ACM ISBN 978-1-4503-6750-9/20/10_..\$15.00

https://doi.org/10.1145/3320269.3384759
}

\section{ACM Reference Format:}

Abhishek Bichhawat, Matt Fredrikson, Jean Yang, and Akash Trehan. 2020. Contextual and Granular Policy Enforcement in Database-backed Applications. In Proceedings of the 15th ACM Asia Conference on Computer and Communications Security (ASIA CCS '20), October 5-9, 2020, Taipei, Taiwan. ACM, New York, NY, USA, 13 pages. https://doi.org/10.1145/3320269.3384759

\section{INTRODUCTION}

Modern systems collect a plethora of personal user information [3], but their growing complexity makes it increasingly difficult to ensure compliance with data protection policies, and to avoid widelypublicized data breaches [20]. Application-wide policy compliance checks are a widely-used way to address these concerns, but ensuring correct enforcement across component boundaries in applications is particularly challenging because it requires coordinating such inline checks across different parts of the application. Thus, enforcing compliance in applications and updating them with new regulations, as they are introduced, is time-consuming, expensive, and error-prone.

The policies that regulations often require, and that these applications need to enforce, include more than just access conditions - they specify how the data can flow and be used by different users, and are contextual or context-dependent. Some other policies might require declassification, revealing partial information about sensitive data in certain cases. Both contextual and information release policies are prevalent in major privacy laws [17, 32, 45] and organizational policies [13, 19, 28].

\subsection{Motivation}

We illustrate the need for supporting contextual and release policies using the example of the Health Insurance Portability and Accountability Act (HIPAA) privacy rule [32].

H1. While some sections of HIPAA restrict the use or disclosure of protected health information (PHI) for marketing or research, other sections allow disclosure if the patient is in need of emergency treatment. Such contextual policies depend on the setting and circumstances of data access and use, i.e., sensitive data may need to be retrieved differently, or disallowed, depending on the conditions under which access is needed (e.g., purpose of access). 
As it is essential that in case of an emergency a doctor in the emergency department must be allowed access to the PHI of the patient, normal authorization rules might not apply.

H2. HIPAA also includes declassification policies - for instance, hospitals are allowed to release aggregate information like the total number of patients diagnosed with a particular disease to be released as it is not PHI (although derived from it), but the individual details of affected patients may not be revealed. For declassification policies, the same set of data accessed individually and together must be subject to different policies.

The main challenge in specifying and enforcing such policies lies in the fact that doing so may require detailed information about the application's internal state and architecture, as well as the underlying database (whether the data is accessed for emergency or regular treatment, or is it a part of some aggregate information or is individually identifiable).

There are numerous existing options for enforcing data protection policies in database-backed applications. Most enterprise database systems incorporate role-based fine-grained access control mechanisms $[23,40,41]$ that can enforce policies that prohibit specified users and groups from accessing certain tables, rows, columns or cells, or provide policy-specific views of the database to different roles. However, because these policies cannot refer to information about the applications that use the database, they are not sufficient for enforcing the contextual policies needed by many applications, and may introduce performance overheads when propagating updates for simpler policies [27].

Several instances of prior work $[6,26,27,35,44,46,47]$ have explored dynamic query-transformation or query-rewriting methods to enforce different sorts of policies, but these approaches do not immediately address the challenge of managing context and application state, and do not support partial release policies that require modifications to query responses. For instance, in the emergency access example $(H 1)$, some of the proposed approaches $[6,16,26]$ include additional conditions like purpose with the traditional access control mechanisms where the user specifies the purpose of access additionally, which is then used to determine access. This, however, requires the electronic health record systems (EHRs) to trust the user requesting access who may not always be benign [25]. Other EHRs might provide a single access point that always allow access to the data, and use after-the-fact auditing for ensuring compliance.

\subsection{Goals, Insights and Contributions}

A proper solution to enforce policies in database-backed applications to ensure compliance with data protection laws and regulations should meet the following criteria in addition to supporting basic cell-, row- and column-level access policies:

(1) Contextual data flow policies: The enforcement mechanism should be able to enforce different policies based on the context of data access or the current state of the application.

(2) Granular policies for complete mediation: Apart from supporting cell-, row- or column-level policies, the enforcement should be amenable to providing partial data disclosure, which can also modify sensitive data before disclosure.
(3) Enable a clean separation between the policy specification, the application, and its underlying database: Given the scale of modern applications, enforcing policies as inline checks can quickly become difficult to maintain and implement correctly. Thus, it is imperative that the enforcement framework should be independent of the application and the database.

(4) Practicality: As a separate policy enforcement mechanism incurs overhead for policy selection and application, it should be a practical solution for the users offering very low or negligible performance overheads while correctly enforcing the policies.

In this work, we build on two key insights for enforcing contextual and granular policies:

1. Application-specific information needed to enforce fine-grained contextual policies can often be obtained at the API-endpoint boundaries of such applications: Typically, servers expose endpoints that a client can call into using a uniform resource identifier (URI). These endpoints map to different APIs defining distinct functionalities, which can further be mapped to what data is retrieved from the database and under what context the access occurs. As different APIs may access the same data from a database under different contexts, policies that are API-specific will reflect the necessary application context. For example, if a doctor in the emergency department of a hospital requests access to a patient's PHI through the emergency treatment endpoint (say, /emergency/), it can be reasonably inferred that the purpose of data access is an emergency. Thus, the context of data access can be determined based on the API endpoint used to access the data. Even if there isn't such a distinction of access, the application code can be refactored to introduce this distinction so that the application can correctly specify the policies.

2. Policies that selectively release information can be applied after query evaluation by mediating the results returned by the database: While normal access policies can be applied before data access by query modification, policies that modify the content of the data cannot be enforced via query rewriting (when dealing with multiple policies - more in Section 3.4). Thus, a declarative policy specification in SQL is inadequate to enforce policies that release parts of a cell's content. However, such policies can be enforced by modifying the data returned by the database after query evaluation.

We present Estrela, a policy enforcement framework that addresses the challenge of enforcing contextual and granular policies for database-backed applications. In Estrela, developers specify policies that are explicitly associated with specific APIs provided by their application. The policies are associated with the database schema separately from the application code without requiring any support from the back-end database. This makes it easier to implement, modify, and audit both the policy and application.

Estrela supports fine-grained contextual policies that are factored into pre-eval and post-eval components. Database accesses throughout the application are subject to pre-eval policies, which are enforced before database access by query rewriting. Post-eval policies provide the flexibility needed by partial release or declassification requirements, by modifying the results of a database query at the granularity of individual rows. 
We demonstrate that Estrela can be applied to real applications to enforce a diverse range of practical data access policies. We prototyped Estrela in Python, on top of Django, and applied our prototype to migrate and build applications with complex policy requirements: a port of the open-source Spirit forum software [43] and open-source social-networking site Vataxia [36], modified to enforce fine-grained data flow policies that restrict how users access topics, posts and user-profiles; a conference management system built on top of Jacqueline [52] used for an academic workshop ported to Estrela; and as a microbenchmark, we build an intranet application that manages the profile and compensation details of employees, and facilitates events and meetings. Using these applications, we show that Estrela incurs very low overhead over the original applications where policies are inlined through the code.

1.2.1 Contributions. To summarize our contributions, we present a novel policy enforcement framework, Estrela, that supports rich contextual policies on sensitive data while maintaining strong separation between policy, code and data. Estrela factors policies into pre- and post-eval components, enabling both data access and information release restrictions in policies. Estrela does not require modifications to the application or the database, thus simplifying policy implementation and maintenance. We prototype an enforcement mechanism for Estrela policies as an integrated language runtime in Python on top of Django and evaluate it by using it to build/migrate four applications ranging from $1000 \mathrm{LOC}$ to $80 \mathrm{kLOC}$. We show that it incurs low overheads while requiring minimal changes to existing application code.

\section{ESTRELA OVERVIEW}

Estrela is a framework that assists the developer in building policycompliant applications. In Estrela, a policy is specified centrally alongside the database schema, and the runtime ensures that the policy is enforced correctly across all application components. Estrela supports fine-grained, contextual policies that are factored into pre-eval and post-eval components. Policies are enforced based on which tables and fields (and their transformations) are queried.

\subsection{Policy specification in Estrela}

Policies in Estrela have the following form:

$$
\mathrm{t}_{1} \cdot \mathrm{f}_{1}, \ldots, \mathrm{t}_{n} \cdot \mathrm{f}_{n} ; \varphi ; \mathbb{A}: \mathbb{P}
$$

In this expression, $\mathbb{P}$ is the policy body containing executable code that either rewrites a query or filters a set of results; $\mathbb{A}$ is an optional API identifier; $\varphi$ is either pre or post; and $t_{i} . f_{i}$ is a column identifier from the target database schema.

The policy $\mathbb{P}$ applies when $\mathrm{t}_{1} . \mathrm{f}_{1}, \ldots, \mathrm{t}_{n} . \mathrm{f}_{n}$ are accessed in a query. $\varphi$ indicates whether the policy is a pre-eval policy (pre) or posteval policy (post) depending on which the policy is applied before or after the query is executed. The function $\mathbb{P}$ works on either an unevaluated query for pre-eval policies, i.e., before the data is actually fetched from the database, or rows in the result-set of an evaluated query for post-eval policies. $\mathbb{P}$ always returns a modified query or row, respectively.

Pre-eval policies: Database accesses throughout the application are subject to pre-eval policies, which are enforced before query evaluation by modifying queries to have additional conditions. These policies add additional filters to the query by either adding conditions to the query's WHERE clause or inserting subqueries, thereby limiting the information returned by the database and the rows being accessed by the query.

Post-eval policies: Estrela supports post-eval policies that provide the necessary flexibility by associating policies with the result of an evaluated query. Such policies operate at a finer-granularity with more contextual information, and are used alongside generic preeval policies for complete mediation. They are mainly information release policies that apply on the query's result, and modify the rows in the result of the query or the result itself. Only the field(s) for which the policy is specified is(are) modified while other fields in the result remain unchanged.

Policies can additionally be associated with a transformation function on a field, e.g., Avg, in which case one of the $t_{i} . f_{i}$ is replaced with $\mathrm{F}_{i}\left(\mathrm{t}_{i}, \mathrm{f}_{i}\right)$ where $\mathrm{F}_{i}$ is the transformation used on $\mathrm{t}_{i} . \mathrm{f}_{i}$.

The field $\mathbb{A}$ contains the list of APIs on which the current policy applies. Based on the API through which the data is accessed, the policies with $\mathbb{A}$ take precedence and override other policies. If $\mathbb{A}$ is omitted, then the policy is applied for every access.

The policies, additionally, have access to the current user authenticated with the system and the API that made the query to the database, which are extracted from the request sent to the server from the client. The current user is represented as $\mathbb{U}$ in the policies. If the user is not authenticated with the server, the user is treated as an anonymous user. The policy selection algorithm checks if the current API is present in the $\mathbb{A}$ field of the policy and returns the policies that apply for that API.

In case policies are specified for $t_{1} . f_{1}, t_{2} . f_{2}$, and $t_{1} . f_{1}, t_{2} . f_{2}$, all three policies are applied when accessing $t_{1} \cdot f_{1}, t_{2} . f_{2}$. If no policy applies on a query, the default policy returns no rows to account for missed policies.

\subsection{Estrela through Examples}

Using an intranet for a large organization as a running example, the remaining section describes example Estrela policies and their specifications. We start by showing simple access control policies followed by how more complex policies are enforced, as the section proceeds. The intranet provides different services to employees like viewing personal employee details, payroll information, and setting up meetings and events within the organization. The schema for the back-end database of this application is shown in Table 1. Briefly, the User field contains the personal details of the company's employees; the Payroll field stores the details of employee salaries along with their manager's identifier; the EventCalendar field records the events or meetings organized within the company while Invitee stores the list of employees invited to each event.

In the examples that follow, we use an object-oriented policy specification that employs pre-defined methods (e.g., filter, exclude) to specify policies for enforcement on SQL queries. Evaluation of a query executes it on the database and retrieves the relevant rows as the result. The pre-eval policies work on a query (query in the policy) and are applied before the query is evaluated. On the other hand, the post-eval policies apply on the result of evaluation of a 
queryset (result in the policy). Policies have access to the current user authenticated with the server (denoted $\mathbb{U}$ in the policy).

Example 1 (Access control policies): Suppose that the site enforces a policy that allows either the name or the age of the employees to be accessed separately, but data linking the name and the age should only be accessible to the employee whose name and age is being accessed, or to an employee from the HR department. This is a basic access-control policy that defines what data is accessible by a user in terms of the database state, and can be specified as a pre-eval policy in Estrela. The annotation pre in the first line of the policy that follows the list of columns on which the policy applies, identifies the policy as pre-eval, as shown below:

User.name, User.age; pre :

$$
\begin{aligned}
& \text { if } \mathbb{U} \text {.dept ! = 'HR': } \\
& \quad \text { query }=\text { query.filter }(\mathrm{id}=\mathbb{U} . i d) \\
& \text { return query }
\end{aligned}
$$

The policy above checks if the current user (identified by $\mathbb{U}$ ) is in the HR department. If so, the policy doesn't add any filters to the original query. If not, the policy ensures that the user accesses only his/her own record. The variable query refers to the query object that will be executed on the database while the function filter adds additional constraints on the query. In the above example, the function adds a clause to remove those rows whose id is not equal to the current user's id.

In certain cases, instead of denying access entirely, the application needs to release some information about a sensitive datum such as an aggregate statistic or a derived value. These policies can be expressed as pre-eval policies in Estrela. In our running example, suppose that the policy requires that non-manager employees can only access the average for other non-manager employees:

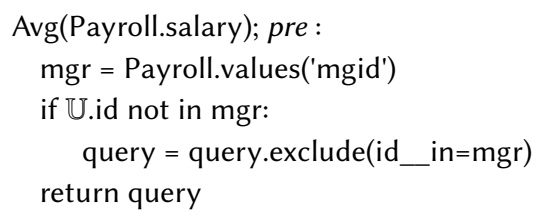

The above policy is enforced when the average of employee salaries (Avg(Payroll.salary)) is accessed by an employee. The policy, initially, retrieves the employee-ids of all managers in the organization. The function values returns only those fields in the table (in this case, Payroll) that are specified as an argument to the function, i.e., mgid (the manager's id). If the current user is a manager, it returns the average of salaries of all employees. Otherwise, it adds a clause to remove the salaries of employees who are also managers in the query, before computing the average, using the exclude function.

\begin{tabular}{|l|l|}
\hline \multicolumn{1}{|c|}{ Table } & \multicolumn{1}{c|}{ Fields } \\
\hline User & id, name, age, address, dept \\
Payroll & id, mgid, salary \\
EventCalendar & eid, date, location, orgid, event \\
Invitee & eid, empid \\
\hline
\end{tabular}

Table 1: Database schema for a company's intranet
Example 2 (Context-dependent partial release): There are other cases where the developer might want to release partial information about the sensitive information, which might vary according to the context. In Estrela, these policies are most naturally expressed as post-eval policies that are applied to a set of query results using the result object. This is denoted with the post annotation at the top of the policy.

Suppose the application enforces a policy that the address of an employee is selectively visible to other employees, such that only the employee can see his/her complete address, employees in the Transportation department can see the neighborhood of an employee to arrange a drop-off, and all other employees can only see the city name. As pre-eval policies apply on the query and not on individual rows, it is not possible to perform different transformations on different rows. The policy given below demonstrates how a post-eval policy can post-process results to enforce this finer constraint:

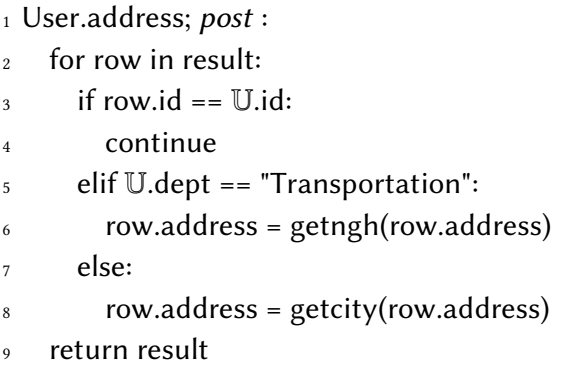

This example returns different values based on the context. If the current user is the employee itself, then the user is allowed access to the complete address (line 3). If the user is in the Transportation department (line 5), the user can see the neighborhood of the employee's address. If none of the two conditions hold, the user sees only the city of the employee.

Example 3 (API-specific differential access): A more interesting scenario arises when the developer wants to specify a policy that indicates only the existence of a sensitive value in certain cases, and in other contexts reveals more information. Such policies are not supported by any of the existing mechanisms. An example of this is a calendar application, wherein the app allows users to create new events and invite other employees to the events.

Consider three endpoints identified by APIs get_events, delete_events and get_location_events shown in Listing 1. The API get_events returns a list of events, the API delete_events returns a list of events that can be deleted, and the API get_location_events returns the list of events organized at the location identified by loc. The function all retrieves all rows from the table. The system enforces a pre-eval policy that allows a user to see only those events that the user is invited to. When accessed through the delete_events API, the system enforces a pre-eval policy that allows the user to see only those events that the user created. However, when viewing the list of events at a location, the user besides getting the details of the events to which she is invited should also see "Private event" for other events along with their date and time so that she cannot schedule another event at the same location at the given time. 


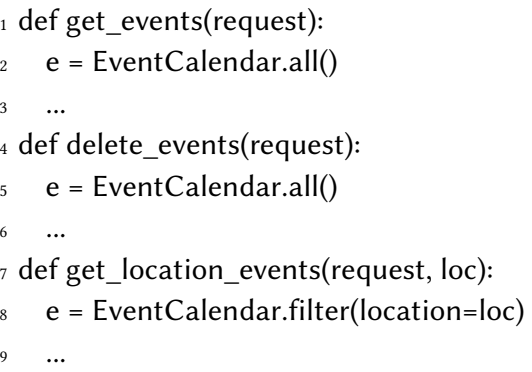

Listing 1: APIs exposed by event calendar service

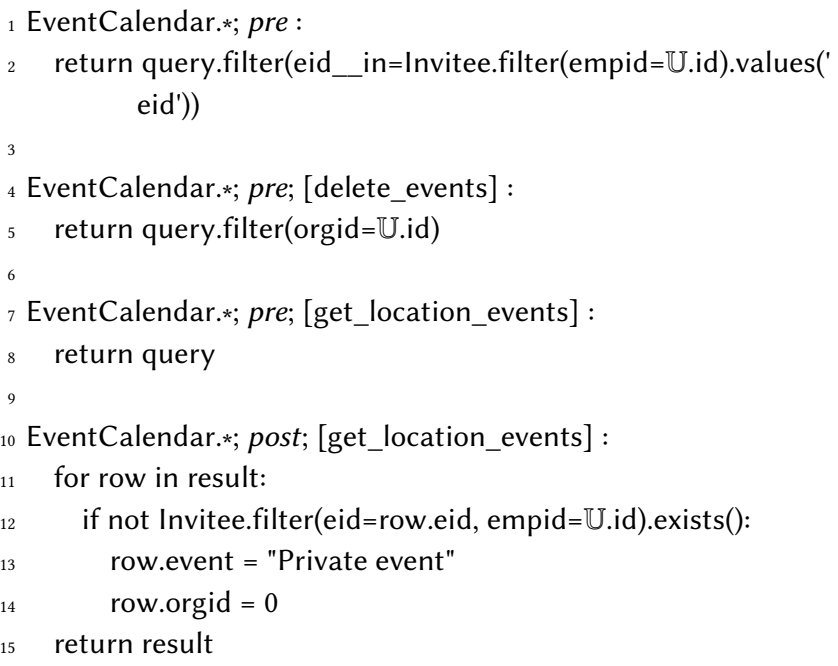

Listing 2: Policies enforced by event calendar service

As getting a list of events is subject to the pre-eval policy that returns only those events that the user is invited to, a policy needs to be specified specifically for APIs accessing events to delete some events, e.g., delete_events, and at a location, e.g., get_location_events. The list of specified policies is shown in Listing 2. For APIspecific policies, the list of APIs that these policies apply to are specified after the pre or post annotation. If the value is omitted, the policy applies to all APIs.

When the list of events is accessed via get_events, it applies the pre-eval policy defined on line 1 and returns only those events that the user is invited to. The ' $*$ ' in the policy indicates that the policy applies every time the table is queried. When the list of events is accessed via delete_events, it applies the pre-eval policy defined on line 4 overriding the policy defined on line 1 as it is API-specific, and returns only those events that the user has created. When the list of events at a given location is accessed via get_location_events, it retrieves all events from the database at that location as the post-eval policy defined on line 10 overrides the pre-eval policy. It checks if there is an entry in the Invitee table that corresponds to the current row's event and the current user (line 12). If found, the policy returns the details of the event to the user; if not, it returns "Private event" for the event (line 13) with the organizer's data hidden. The policy on line 7 ensures that the query is not filtered, thus, returning all events registered at the given location. Without

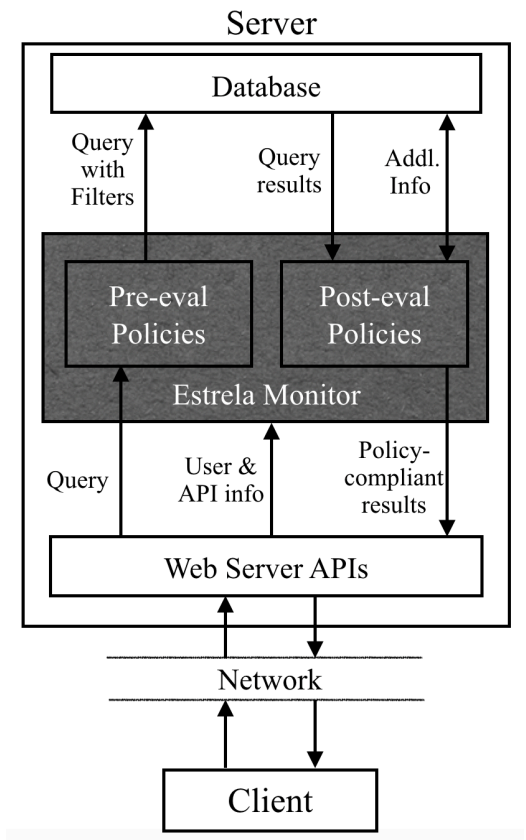

Figure 1: Application architecture with Estrela

this, the policy on line 1 would filter the list to contain only those events that the user is invited to.

\section{POLICY FRAMEWORK}

\subsection{Application architecture with Estrela}

Figure 1 describes the application architecture with the policy enforcement mechanism using Estrela. The overall workflow of the architecture is as follows:

(1) The server accepts incoming requests on different APIs and performs the required query on the database.

(2) Pre-eval policies associated with the data being retrieved are applied as filters on the query before the object is fetched from the database.

(3) The filtered query is then executed on the database, which gets some data from the database.

(4) Once the data is fetched, the post-eval policies that apply on the result of the query modify the query's result-set.

(5) The API performs any necessary computations on the data retrieved, and creates and sends the response to the client.

\subsection{Threat model}

Estrela aims to provide a unified, practical, and robust framework for specifying and enforcing policies in database-backed applications. The primary security goal of Estrela is to ensure that the database queries and the APIs satisfy the conditions specified in the policy. Estrela mitigates authorization bugs by enforcing data flow policies that apply when the data is read from the database, and limit the scope of our paper to those. We assume that developers make a good-faith effort to specify correct policies, and that the integrity of the Estrela framework is intact and uncompromised throughout the lifetime of the application. Estrela does not attempt to prevent 


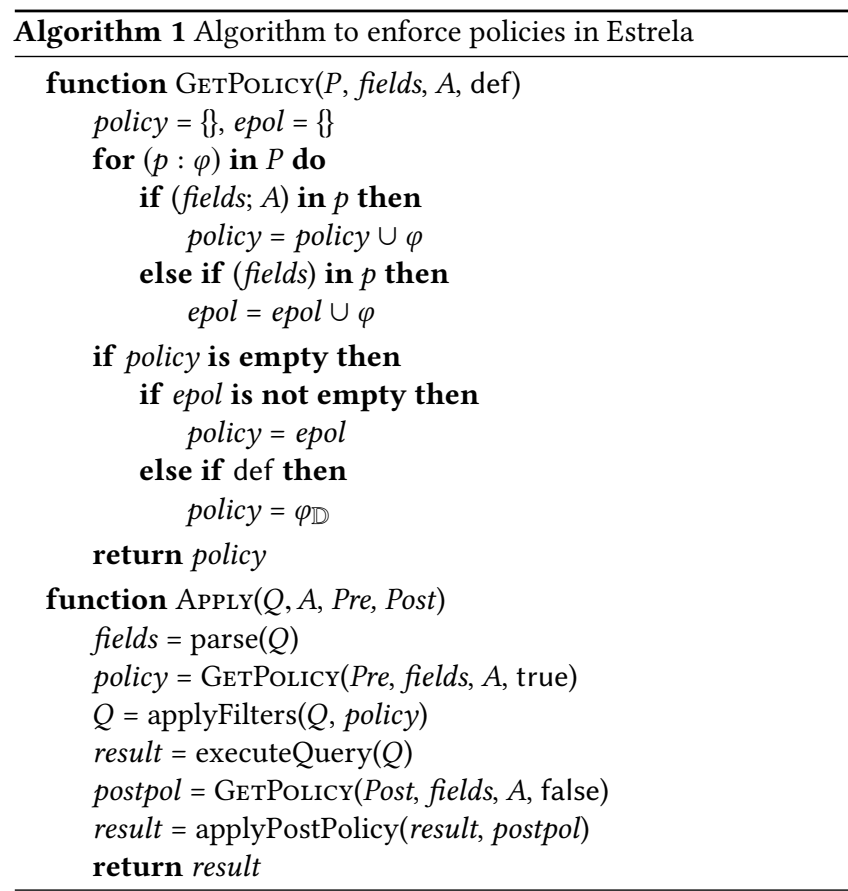

leaks from hardware or operating system-level side channels, or by groups of users who collude via out-of-band channels to learn more than what is specified in the policy. Likewise, as Estrela policies concern server-side data, leaks that result from vulnerabilities in the client-side browser or operating system are also not in scope for our security goal.

\subsection{Algorithm for policy enforcement}

Algorithm 1 shows the top-level algorithm (and the auxiliary function) used by Estrela to select and enforce policies on data access. The top-level function APPLY takes the query, API, and the set of policies as the input. The function starts by parsing the query $(Q)$ to determine which fields are used by the query. Using the fields and the API information $A$, the function GetPolicy returns the pre-eval policies applicable to the query. These policies are enforced on the query, which is then run. The results returned by the database are modified as per the post-eval policies selected by GETPoLICy for the fields and the API $A$. The modified result is then returned to the user.

The operation (fields; $A$ ) in $p$ in GetPolicy checks if the fields and APIs in the policy $p$ contain fields and $A$, i.e., the list of fields and APIs in $p$ is a superset of fields and A. If fields contain aggregate functions, it additionally checks for the fields used in the aggregate function in the fields of $p$. If the appropriate policies for the API $A$ are not found, the function applies general policies that apply to all APIs. The policy $\varphi_{\mathbb{D}}$ is the default policy that restricts access to all the data. The variable def is set to true or false to indicate whether the default policy should apply.

\subsection{Declarative vs. object-oriented policies}

Estrela enforces multiple policies associated with the (set of) fields specified in the query. It filters data based on the pre-eval policies à la the existing access-control mechanisms, and modifies the data in the result to release partial information as per the post-eval policies. In our prototype, instead of specifying policies in a declarative language like SQL, we specify them using object-relational mapping (ORM) methods that offer an object-oriented view and a methodbased access of the data. Besides making it easy to write policy functions for developers who might be more comfortable using object-oriented programming for policy-specification, ORM-based specification has certain advantages over the declarative specification when specifying complex policies:

(1) Post-eval policies cannot be specified in SQL as they apply on a query's result requiring an imperative specification.

(2) Enforcing multiple policies when specified in SQL forces the policies to be access-control (as they are specified as additional conditions in the WHERE clause $[6,26,27]$ or specific SQL sub-queries [26, 47]); thus, not allowing information release unless using user-defined functions that are already defined in the database, which, in turn, requires modification to the application code.

(3) Enforcement of multiple data usage policies for information release (e.g., DataLawyer [46]) require all policies to be checked before the policy-compliant data is returned, making the approach less efficient.

\subsection{Discussion}

3.5.1 Comparison with existing access control enforcements. While the access control mechanisms enforced by enterprise database systems [23, 40, 41] provide fine-grained data access, they have certain shortcomings that make them unsuitable for applying the policies supported by Estrela:

- They lack support for policies that link two or more tables without having explicitly defined views. Creating such views require modifications to the application such that it queries for the correct data.

- Data masking either removes all rows or defaults the values in the column if the rows are inaccessible by the user. They do not support modification of specific rows in the table as shown in Example 2 above.

- Contextual policies are application-specific, and hence cannot be implemented generically by database access control systems. Existing systems $[6,16]$ that take into account such contexts when allowing access require explicit specification by the user in the query, which they validate. While the user needs to be trusted to provide the correct context, it also requires modifications to applications to send appropriate queries.

As most of the other existing approaches [6, 26, 27, 35, 44, 46, 47] work independent of the application, they do not have access to the API-specific information necessary for enforcing contextual policies. It is, however, possible to expose the path or API on which the request is made (similar to how the authenticated user is exposed) to the enforcement monitor. This would, in turn, require the existing approaches to modify the policy specification language to take into account API information for enforcing contextual policies. However, it is not possible to specify post-eval policies in the existing approaches (making modification to query results difficult), 
which is required, for instance, in Example 3 when fetching events at a particular location (get_location_events).

It is also instructive to see how the policy in Example 2 differs from prior work like Qapla [27], which also requires user-defined functions like getngh and getcity for specifying such policies. In Qapla, the developer has to invoke the correct function, e.g., get$\mathrm{ngh}$ (address) if the user is in the Transportation department and getcity(address) if the user does not satisfy any criterion, to get access to the address. If the developer queries the wrong function, she does not get access to the address. In Estrela, the post-eval policy takes care of this; thus no modification to the application or its API is required. Moreover, the query needs to be repeated to get the actual values of address and the city name when the user does not have appropriate access.

Other prior works $[26,47]$ can use CASE statements to specify such properties because their rewriting technique generates different data based on the conditions. However, when multiple policies apply on a query for a column, it is unclear as to how the policies would apply in these approaches.

3.5.2 Pre-eval vs. Post-eval policies. Post-eval policies are at least as expressive as pre-eval policies, but enforcing pre-eval policies has certain advantages. Firstly, as pre-eval policies are applied before the query is evaluated by adding filter conditions or subqueries, the database is queried only once. This enhances the performance of the policy-framework as post-eval policies require more database hits (as the policy queries the database again). Secondly, pre-eval policies prevent timing-related leaks that are possible with posteval policies. As they are applied before the database is queried, they do not reveal any information about the number of records satisfying the original unfiltered query. For instance, suppose, in a healthcare setting, a user wants to know how many patients have a certain disease, but say, they are not allowed to access this information. With post-eval policies, the time taken to first retrieve the list of patients that have a certain disease and then filtering the results might be significant, dominating the time to respond to the query. Therefore, if it takes a long time to respond, this may leak some information on what the query response size was to the user, even though the result itself contains no sensitive information.

Post-eval policies allow partial release of sensitive information based on the context making them flexible enough to handle cases where different values need to be returned as per the context. Moreover, it is not possible to enforce all policies before evaluation as some policies need to post-process the results. Post-eval policies are also useful when some additional information that is not present in the result of the filtered query needs to be released.

3.5.3 Preventing implicit leaks. The policies are applied considering all the fields used in the query irrespective of where they appear in the query. While this may at first seem too conservative, it is necessary to prevent implicit information leaks, as might be the case when, for example a query returns the names of all employees of a particular age (SELECT name FROM employees WHERE age > 45). The policies for accessing name, and name and age together may be different - while all employees can access the names of other employees, an employee can only access his/her own age and name together as shown below:
User.name; pre :

return query

User.name, User.age; pre :

return query.filter $(\mathrm{id}=\mathbb{U} . \mathrm{id})$

If the policy were based only on the selected name column, it would apply a relaxed policy that allows the names of all employees having the particular age to be displayed as the first policy does not apply any filter for access of name. However, as the two columns are linked in the query, the correct policy would be the second one associated with both name and age that reveals to the user his/her own name and age only and no additional information because of accessing name and age together. To handle such leaks, all columns used in the query are considered when selecting the policy to apply on the query.

\section{IMPLEMENTATION}

Estrela provides a mechanism for automatically enforcing a given set of policies. We discuss its implementation in this section and evaluate the approach in Section 5.

Estrela is prototyped in Python and extends Django [15], a Python-based model-view-template application framework. Thus, apps written in Estrela are otherwise standard Django apps with policies in the schema. Policies are specified alongside the database schema using two class-methods (for pre-eval and post-eval policies) that are inherited by all models (schemas). If these methods are not overriden by a model, then a default conservative policy that suppresses all results applies. It includes an object-relational mapping (ORM) to interact with databases based on which it constructs SQL queries, represented as querysets. Evaluation of a queryset object executes the query on the database and retrieves the relevant rows as the result. Django constructs a queryset as soon as an API starts querying for some data in the database; however, it does not evaluate the queryset to reduce the number of database hits. The pre-eval policies work on a queryset (query in the policy) while the post-eval policies apply on the result. Policies are Python functions in our prototype.

To enforce a pre-eval policy, we augment the database interface functions provided by Django via monkey-patching, which amounts to inheriting from Django's classes and overloading the methods relevant to Django's interfacing with the database. We modified about 700 lines of Python code including code from the original implementation. Using this approach, we achieve complete mediation such that all database accesses in an Estrela application occur through the instrumented methods. These methods are responsible for invoking the policy functions, and passing them the current user and API information that Estrela exposes from the request to the server. As Django supports lazy evaluation, we apply the pre-eval policies just before the query is evaluated to get a better performance.

To enforce a post-eval policy, we modify the results returned from the database and selectively apply the relevant policy functions before the results are returned to the APIs. Selective enforcement is achieved by a case analysis on the fields involved in the query, to determine which policies are relevant. When multiple policies apply on the result of an API call, we assume an arbitrary but 
fixed order in which to apply the policy functions to the queryset result.

Both pre- and post-eval policies need to consult the set of active API-specific policies to bypass enforcement when operating in the context of a relevant API.

\subsection{Integration with legacy applications}

As Estrela is built on top of Django, migrating an existing Django application to Estrela is straightforward and does not require changes to the core application code. The developer only needs to specify the policies alongside the data models (i.e., the database schema), which are modified to inherit from Estrela's model class. The only other necessary change is to expose the request parameters of each API to the policy enforcement mechanism, which is taken care of automatically by Estrela using a middleware configured in the application's settings.

Although Estrela is prototyped in Python on top of Django, its principles are generic and can be extended to enforce authorization policies in other existing frameworks (designed using any language). Estrela requires a separation of the models or the database schema from the actual application alongside which the policies are specified in the language in which the framework is built. Enforcing policies would require adding hooks in the query evaluation process of the framework.

An interesting question to consider when integrating Estrela with legacy applications is how to bootstrap policy specification to assist the developers. Policy inference, which is orthogonal to the problem studied in this paper, has been an area of active research where prior works have proposed approaches to mine meaningful policies using logs, traces and program specifications $[1,4,5,11$, $22,24,29,37,48-51]$. The mined policies can be used to bootstrap the initial set of policies when migrating applications to Estrela. However, the problem of policy inference is out of scope of the current paper.

\section{EVALUATION}

We evaluate Estrela by comparing the code changes required on existing applications and the overhead it incurs due to the policy checking. We demonstrate that Estrela is easy to integrate with existing applications, and incurs very low overheads, showing its effectiveness and usefulness. We consider open-source applications for migration to Estrela that are built using Django.

\subsection{Methodology and setup}

We used Estrela to migrate a few applications to enforce policies, ranging from about $1000 \mathrm{LOC}$ to $80 \mathrm{kLOC}$. The first is a version of Spirit [43], a forum software where users can discuss on different topics, migrated to Estrela with policy enforcement. The second application is a social-networking site Vataxia [36]. The third is a multi-user conference management system that lets users add, edit, remove papers for a conference. The fourth application is a company's intranet on the lines of the examples discussed in Sec. 2.

The case-studies were chosen to evaluate the effect of policy enforcement on large applications, applications with multiple policies, and the overhead it incurs on simple and complex policies. For all the case-studies, we add a middleware to expose the request details to the schema for evaluating the policies in Estrela. All other code in the applications remains the same. The baseline implementations of our case-studies are all built using Django with policies included in the code, allowing us to evaluate the performance of Estrela.

We performed our experiments on a MacBook Pro having an 8 GB RAM and $3.1 \mathrm{GHz}$ Intel Core i5 processor, running macOS Catalina 10.15.1. We automated the process of sending requests to the server to retrieve data from the database, and measured the average time taken for processing the request and policy enforcement over 100 trials. The back-end database was MySQL version 8.0.18. Unless mentioned otherwise, all servers were build using Python 3.7.3 and Django 2.2.7. The server and the client run on the same machine so the evaluation results do not include network latency or the time taken for rendering. We ran this process in the context of a particular user, who is authenticated before sending the request. The error bars in the graphs show the standard deviation.

In the following sub-sections, we describe the functionality of these applications, the policies that we apply, and how these policies are implemented and enforced using Estrela. We report the performance numbers for these case-studies, by measuring the time taken by the application to perform user operations in different scenarios. Section 5.2.1 discuss the code changes required for the implementations to enforce policies in their respective settings.

\subsection{Case study: Spirit forum software}

Spirit is a Django-based forum software for facilitating conversations and discussions amongst users. The original software contains about $80 \mathrm{kLOC}$. Users can create new conversations or post comments on existing conversations depending on the visibility of the conversation and/or whether the user has been invited to the conversation or not. The schema of the actual site contains 28 tables with 165 columns. We specified pre-eval policies for various tables and measured the overhead incurred by Estrela, and demonstrate the ability of Estrela to scale to large applications.

5.2.1 Migration effort. We modified the models' base class in the original software to use Estrela's model as the base class. This required a modification of about $30 \mathrm{LOC}$ in the original software. The other modification required apart from the specification of policies was to expose the incoming request to the models for identifying the current user and the API that requested the data, which require a couple of lines of code to be added to the settings of the application.

One of the policy we enforce is associated with various topics in a forum that allows only logged-in users to view the topics. Without the policy enforcement, Spirit shows all public topics even without a user being logged-in to the system. In Estrela, we added a policy that checks if the current user is authenticated or not; if not, it does not show any topics to the user. In the original version of Spirit, this check has to be propagated to at least three different files in the codebase, all of which access a topic and display it to the user reiterating the need for centralized specification of policies.

5.2.2 Performance. We added 1000 users and 1000 topics to the database and evaluate the time taken to access the topics with different users. The result of our experiment is shown in Figure 2. Estrela incurs an average overhead of about $0.2 \mathrm{~ms}$ or $0.8 \%$ when 


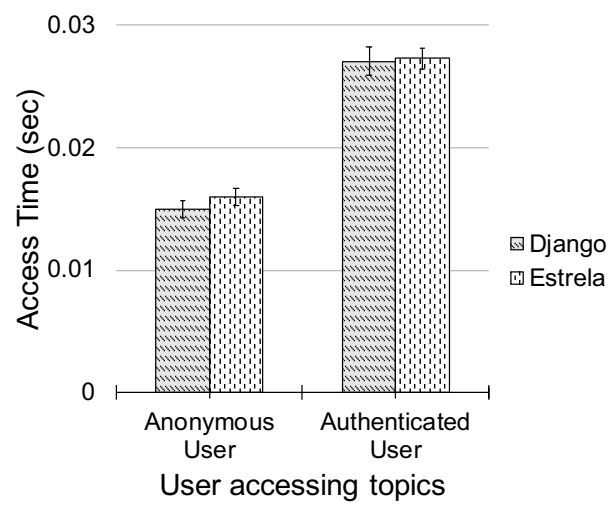

Figure 2: Time taken to request and access different topics in the forum with Django as the baseline

accessing the topics as an authenticated user. When accessing the topics as an anonymous user, Estrela incurs an overhead of about $1 \mathrm{~ms}$, which is mainly due to Django's lazy evaluation in Estrela. With Estrela, the policies are applied just before the evaluation after the objects have be created even if the objects are never required in the future resulting in the additional overhead. The inline check in Django can be added as early as possible in the API code preventing the creation of objects, thereby avoiding the additional overhead.

\subsection{Case study: Social-networking site}

Social-networking sites involve multiple users posting information and sharing it with other users. Users can track the activity of other users by "following" them. We modify an existing open-source social-networking site, Vataxia [36], by implementing additional functionality to follow users and view a user's posts. The application allows new users to be added to the system and to search for users to follow their posts. The original schema contained the following models - User, Post, PrivateMessage, Reply and Vote; we extend User by adding a field, follow, to include a list of users that the user is following. The table Post contains a field user referencing User table, and the message that the user has posted in msg. The front-end of the application is written in ReactJS while the back-end is developed using Django REST framework [14], Django 2.2.7 and Python 3.7.3.

5.3.1 Migration effort. We port Vataxia to Estrela by adding policies for various tables modifying the models of the application to inherit from Estrela's base class. The original application contains around $19 \mathrm{kLOC}$ as part of the front-end and $2 \mathrm{kLOC}$ in the back-end. We modify 10 lines in the application, and include the middleware that exposes requests to the models.

We discuss the enforcement of a policy that limits the posts that a user can view based on whether the user is following the user whose posts are being accessed or not. If accessing only the posts, a user can see posts by only those users that she is following. However, when the user accesses a profile of a particular user, she gets a default message asking her to follow the user to see the posts. In Estrela, this policy is implemented as a post-eval policy specific to the profile_view API because when accessed through the posts_view API, this post-eval policy should not be enforced.

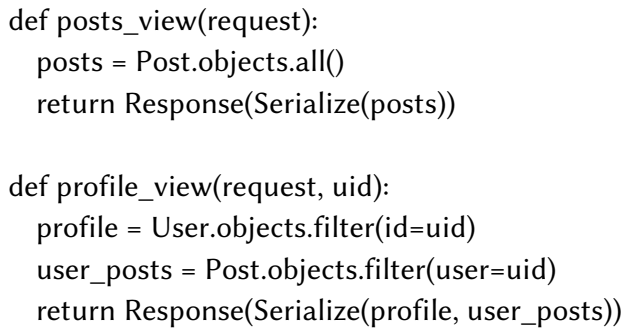

Listing 4: Policy specified in Django for Vataxia

Post.*; pre:

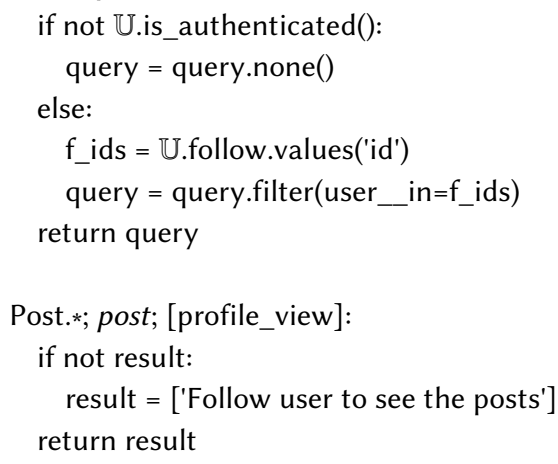

\section{Listing 5: Policy enforcement for Vataxia in Estrela}

For code comparison, the policy codes for Vataxia are shown in Listings 4 and 5 for policy in views, and schema with Estrela, 


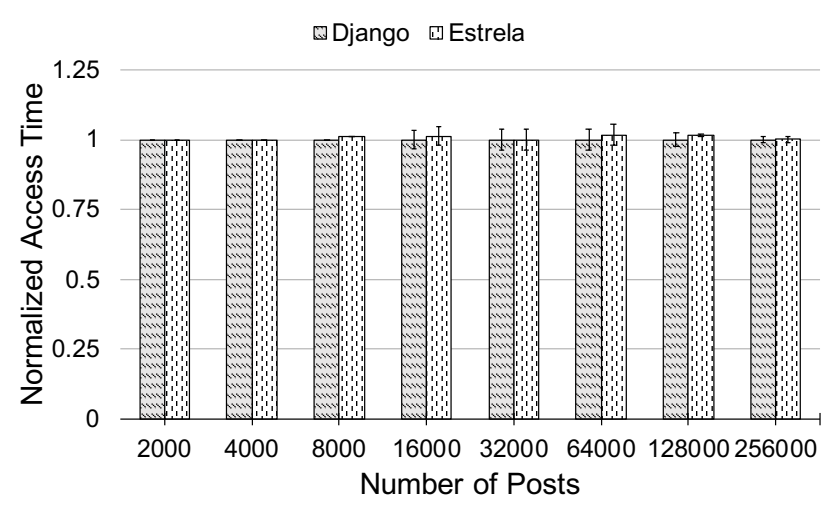

Figure 3: Normalized time taken to access all posts through a REST API with policy in code as the baseline

respectively. Although the policies are similar across the two Listings, the developer has to add the policy for every access of Post when adding checks in the API code (e.g., on line 7 and line 18 in Listing 4) while the policy specification is much cleaner and does not require any modification to the code in Listing 3.

5.3.2 Performance. We added 1000 users and their details to the database for the social-networking site with every user following $0-3$ other users in the system. We measure the time taken to access posts in the system for different number of posts (ranging from 2,000 posts to 256,000 posts). The posts are generated automatically before the processing starts and are related to random users. Figure 3 shows the normalized time taken in different scenarios. The average overhead for accessing all posts with Estrela when compared to the baseline of policy in the application code is around $0.7 \%$. The policy in Estrela applies on the result before it is returned, but incurs the overhead due to the additional check for authentication of the user and selecting policies; the enforcement time for the policies is almost the same as with policies intertwined with the code.

\begin{tabular}{|c|l|}
\hline Table & \multicolumn{1}{c|}{ Policy - User can access } \\
\hline User & $\begin{array}{l}\text { either her own profile or another user's profile if she } \\
\text { is the chair or in the pc } \\
\text { any paper if she is the chair } \\
\text { non-conflicted papers if she is in the pc } \\
\text { Paper } \\
\text { Paper } \\
\text { Paper } \\
\text { accepted status of a paper either if she is the chair, or } \\
\text { if she is the co-author and phase is final } \\
\text { number of submitted and accepted papers in final } \\
\text { phase }\end{array}$ \\
\hline
\end{tabular}

Table 2: Policies for the conference management system

\subsection{Case study: Conf. management system}

We modify an existing conference management system built in Jacqueline [52], using Django, that has been deployed to manage paper reviewing process for an academic workshop (PLOOC 2014), to work without information flow tracking in place and migrate it to Estrela. This modified application contains about $4 \mathrm{kLOC}$ retaining the features of the existing system like creating users and

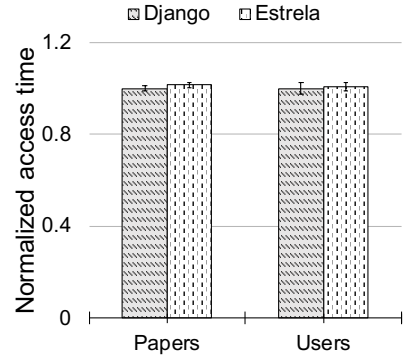

Figure 4: Normalized time taken to access all papers and users with Django as the baseline

conferences, adding papers and roles for users, etc. while removing its dependence on the Jeeves [53] library and the functionality to handle faceted values; the database also does not contain additional fields for handling facets. The system supports single-blind submission, handling conflicts, and submission of reviews and comments by reviewers. Every conference has three phases - submission, review and final - which influence the policies. We enforce the policies shown in Table 2 on different tables in the schema.

5.4.1 Performance. We created a dummy conference with 1000 users ( 25 of them on the pc), and 1000 papers submitted by randomly chosen authors with 2 co-authors each. The policy for User table shown in Table 2 is a pre-eval policy while Paper has a mix of both. Figure 4 shows the normalized time taken to access all papers and users in the system with Django as the baseline. The performance of Estrela when accessing the list of users incurs an overhead of about $0.6 \%$. Estrela incurs an additional overhead of around $1.5 \%$ as compared to Django when accessing the list of papers due to the number of policies (both pre-eval and post-eval) associated with the Paper table.

\subsection{Case study: Company intranet}

As a microbenchmark to evaluate the performance of Estrela when enforcing different kinds of policies shown as examples in Section 2, we implement an intranet website in Estrela that handles employees' personal and official information. The employees of the company can access their profiles, payroll information, their friends' profiles, and can schedule events or meetings within the company as described earlier in Section 2 extended with an additional Friends table that contains a list of friends in the organization. A brief description of the policies that we enforce is shown in Table 3. The policy-compliant queries executed on the database through Django and Estrela are the same. As policies in Estrela use high-level functions that are also used for the inline checks in Django, the modified query generated in Estrela is the same as the one generated through Django using inline checks.

5.5.1 Performance. For the intranet example, we added employee details for 20,000 users in the database with each having at least 5 friends. Additionally, we added 100000 events to test the scenarios involving events (Q5 and Q6). The policies for Q4 and Q6 are posteval policies while for the other examples are pre-eval. We measure the time taken to access employee and event information for the six examples shown in Table 3 . The normalized execution time 


\begin{tabular}{|c|l|}
\hline Query & \multicolumn{1}{c|}{ Policy - Employee can access } \\
\hline Q1 & $\begin{array}{l}\text { only her friends' ages } \\
\text { all employee details if she is in the HR department; if } \\
\text { not, only her own details } \\
\text { average salary of employees who are not managers } \\
\text { unless she is a manager }\end{array}$ \\
Q4 & $\begin{array}{l}\text { address of her friends but sees only the city name for } \\
\text { other employees } \\
\text { only those events to which she is invited } \\
\text { events at a particular location but sees "Private event" } \\
\text { for events to which she is not invited }\end{array}$ \\
Q5 \\
\hline
\end{tabular}

Table 3: Microbenchmark - Policies enforced for the intranet website

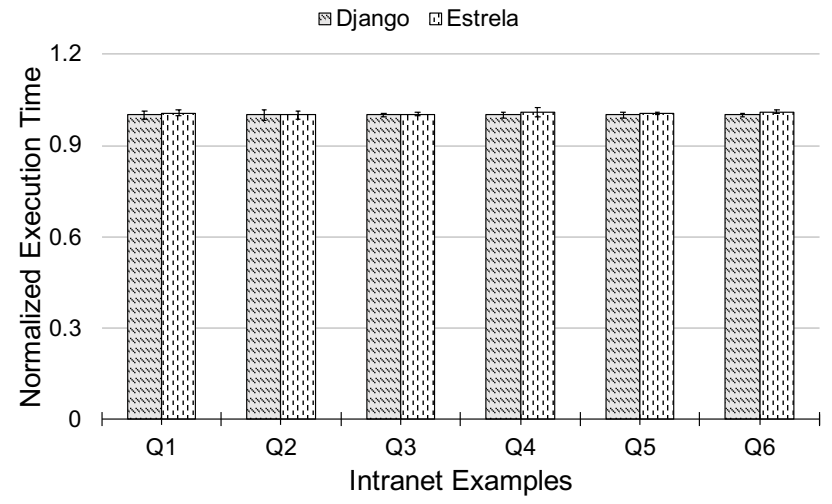

Figure 5: Normalized execution time for intranet examples with Django as the baseline

for the examples with policies enforced in Django and in Estrela is shown in Figure 5 with Django being the baseline. The time includes the time taken to send the request, apply the policy, execute the query, and to send back the response to the client. The overhead for policy enforcement in Estrela ranges from about $0 \%$ when accessing employee details to $1 \%$ when accessing a set of events at a particular location. The additional overhead for Estrela is due to the checks to select the correct policies to apply.

Throughput. To examine Estrela's impact on the throughput, we measured the number of requests handled per second when issuing the queries Q1 - Q6 described above. We used ApacheBench version 2.3 to measure the throughput with a concurrency level of 10. Each run issued 500 requests. Figure 6 shows the resulting degradation in throughput, which was around $1 \%$ in the worst-case.

\subsection{Policy complexity}

We show in Figure 7, how the complexity of a policy affects the overhead of Estrela. We create an application in Estrela with a couple of tables each having 100 columns and a 100,000 rows, and test the performance for different number of columns being used in the policy. We ensure that all conditions in the policy are evaluated to get the worst-case results, and evaluate it for both - Django with policy in the API code, and Estrela. The overhead that Estrela adds

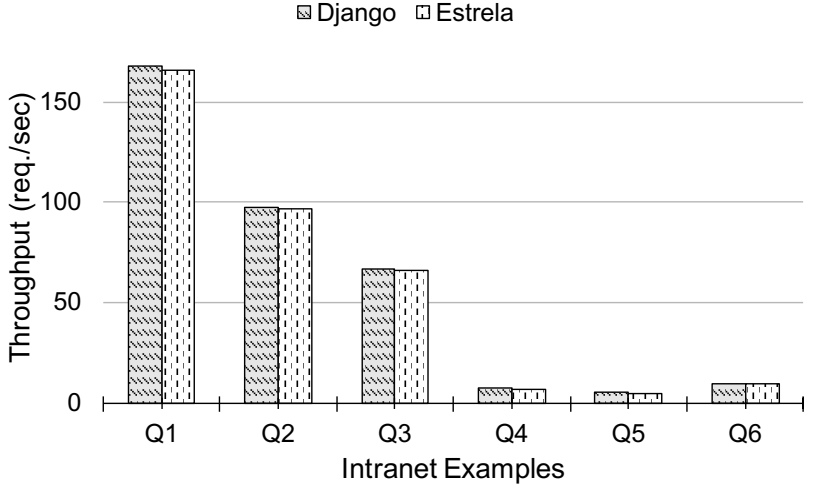

Figure 6: Throughput of Estrela and Django for the intranet application with a concurrency level of 10

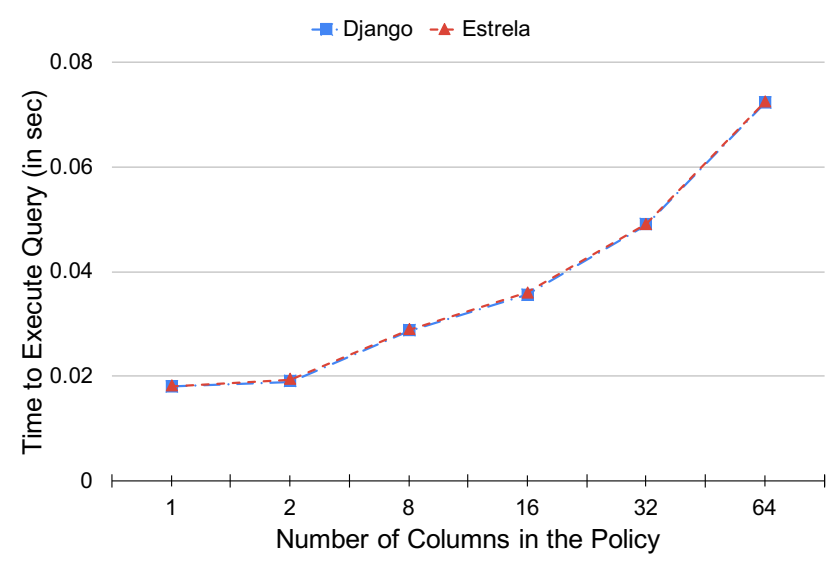

Figure 7: Time taken for executing a query subject to a pre-eval policy with different number of columns

is almost constant, and does not increase as the complexity of the policy increases.

\section{RELATED WORK}

We describe some of the closely related works to Estrela on policy enforcement, most of which deal with enforcing policies on the server-side of applications that communicate with a database to retrieve and store sensitive data.

Qapla [27] is a framework to provide fine-grained access control in database-backed applications where the policies are specified as SQL WHERE clauses that define what information the users are allowed to access. Qapla's enforcement engine modifies the low-level SQL queries made to the database by adding the policies as sub-queries. However, it does not support the specification of default values that reveal the existence of a sensitive value, and does not provide the flexibility of applying release policies except aggregation offered by the post-eval policies in Estrela. Extending Qapla to support post-eval policies is non-trivial because such policies cannot be specified as SQL WHERE clauses requiring an additional policy specification mechanism. Moreover, integrating Qapla with existing applications might require modifications to the 
application code to query for the correct column-transformations without which it returns a more restrictive set of results.

Hails [18] is an MPVC web framework that adds policies declaratively alongside database schemas and tags every piece of data in the system with a label. These labels are carried around as the data flows in the system and checked by a trusted runtime when leaving the system. The focus is to control the flow of data to untrusted third-party applications by building applications using the Hails web platform. Similarly, Jacqueline [52] is a framework to track information flow in applications dynamically using the policyagnostic design paradigm. Jacqueline relies on a modified database that stores multiple views of data based on who is allowed to access what. It additionally allows specification of default values, but does not support policies that are linked to a set of sensitive fields in the database or policies involving data-aggregates. While Estrela primarily enforces server-side policies without any client-side information flow tracking, it supports contextual policies unlike Hails and Jacqueline without requiring modifications to the database. Estrela can be integrated with these frameworks for information flow tracking at the client-side to ensure that the sensitive data does not flow to unauthorized parties.

FlowWatcher [30] is a system that enforces information flow policies within a web proxy without requiring modifications to the application. However, it is difficult to enforce fine-grained policies, like the ones Estrela supports, in the system. LWeb [33] is another system that provides information flow control for web applications developed in Haskell, which supports expressive policies albeit with moderate overheads. LWeb, however, does not support contextual policies and requires building all applications from scratch. Similarly, Daisy [21] provides flow tracking in databases supporting features like triggers and dynamic policies. However, their monitor supports only policies that can be expressed in the SQL access control model. SELinks [10] allows both server-side and databaseside enforcement of policies by compiling server-side functions to user-defined functions that can run on the database for compliance. It does not support contextual policy compliance and is limited to controlling data disclosure.

Sen et al. [42] propose Legalease language for stating policies using Deny and Allow clauses and enforce it on big data systems. The language is simple allowing easy policy specification by the developers and does partial information flow tracking to catch violations of policies, but can express only a smaller subset of policies than Estrela. For instance, Legalease cannot return parts of sensitive information unless stored explicitly in the database and queried explicitly for by the application.

$\mathrm{Ur} / \mathrm{Web}[8]$ is a domain-specific language for programming web applications, which includes a static information flow analysis called UrFlow [7]. Policies are expressed in the form of SQL queries and can express what information a particular user can learn but the analysis might over-approximate being static in nature. DataLawyer [46] is a system to analyze data usage policies that checks these policies at runtime when a query is made to the database. The policies are specified in a formal language based on SQL. It allows quite expressive policies but checks all policies whenever the database is queried. Estrela, on the contrary, selects the right set of policies based on the fields used in the query, making it more efficient. CLAMP [34] protects sensitive data leakage from web servers by isolating user sessions and instantiating a new virtual web server instance for every user session. The queries are restricted to data accessible by the current user; however, the granularity of policies is limited to per-table.

Byun and $\mathrm{Li}$ [6] and later Kabir et al. [16] proposed purposebased access control to enforce purpose-based policies like the ones related to HIPAA showed in the paper. While purpose-based access control takes contextual purpose into account, the existing approaches rely on user's trustworthiness to specify the purpose for access. In contrast, our work uses path-specific information to determine the purpose dynamically.

SIF [9] is a framework for developing web applications that respect some confidentiality policies. The framework is built on top of Jif [31], an extension of Java with information flow control, and enforces information flow control in Java Servlets. Besides being language-specific, SIF also incurs moderate overheads because of the analysis. SeLINQ [38] is another information flow control system to enforce policies across database boundaries that modifies a subset of $\mathrm{F} \#$ with database queries to perform information flow analysis. IFDB [39] enforces information flow control in databases in a decentralized fashion by associating labels with every data object. It uses specialized views for pre-eval policies. RESIN [54] allows developers to check data-flows in the program using assertions, which are checked at sinks in the program without developer-added checks. It can also express rich policies as assertions in the program. Nemesis [12] focusses on preventing authentication and access control vulnerabilities in web applications by using dynamic data flow tracking. These systems label information and track its flow through the application and guarantee that information flows as per the policies but are specific to the respective languages.

Passe [2] allows enforcement of learned security policies by running applications as a set of isolated or sandboxed OS processes and restricting what data each process can access or modify. The principle behind Passe is to separate the privileges of various parts of an application as per its requirements and granting least privilege access, as required, for running the isolated parts. The constraints are discovered in a learning phase inferring the data-flows and control-flows in the application to limit the flow of data. Estrela, on the other hand, associates developer-specified policies with APIs.

\section{CONCLUSION}

We present Estrela, a framework that ensures policy-compliance in database-backed applications by supporting specification and enforcement of contextual and granular policies. The policies are specified separately from the application code alongside the database schema without requiring any modifications to the existing code and the database. We prototyped Estrela in Python on top of Django to specify and enforce API-specific policies. Estrela supports easy migration of legacy applications for policy-compliance. We show the applicability of Estrela by building/migrating four applications on top of it and showing that it incurs low overheads while enforcing expressive policies.

\section{ACKNOWLEDGMENTS}

We would like to thank our shepherd, Qi Li, and the anonymous reviewers for their insightful comments and feedback. The work 
was supported in part by the Center for Machine Learning and Health (award no. PO0006063506) at Carnegie Mellon University.

\section{REFERENCES}

[1] Lujo Bauer, Scott Garriss, and Michael K. Reiter. 2011. Detecting and Resolving Policy Misconfigurations in Access-Control Systems. ACM Trans. Inf. Syst. Secur. 14, 1, Article 2 (June 2011), 28 pages.

[2] A. Blankstein and M. J. Freedman. 2014. Automating Isolation and Least Privilege in Web Services. In 2014 IEEE Symposium on Security and Privacy. 133-148.

[3] J. Brookman. 2019. Data Collection Practices of Large Online Platforms. https://www.ftc.gov/system/files/documents/public_events/1415032/ privacycon2019_justin_brookman.pdf.

[4] Thang Bui, Scott D. Stoller, and Hieu Le. 2019. Efficient and Extensible Policy Mining for Relationship-Based Access Control. In Proceedings of the 24th ACM Symposium on Access Control Models and Technologies (SACMAT '19). Association for Computing Machinery, New York, NY, USA, 161-172.

[5] Thang Bui, Scott D. Stoller, and Jiajie Li. 2017. Mining Relationship-Based Access Control Policies. In Proceedings of the 22nd ACM on Symposium on Access Control Models and Technologies (SACMAT '17). Association for Computing Machinery, New York, NY, USA, 239-246.

[6] J. Byun and N. Li. 2008. Purpose Based Access Control for Privacy Protection in Relational Database Systems. The VLDB fournal 17, 4 (July 2008), 603-619.

[7] A. Chlipala. 2010. Static Checking of Dynamically-varying Security Policies in Database-backed Applications. In Proceedings of the 9th USENIX Conference on Operating Systems Design and Implementation (OSDI'10). 105-118.

[8] A. Chlipala. 2015. Ur/Web: A Simple Model for Programming the Web. In Proceedings of the 42nd Annual ACM SIGPLAN-SIGACT Symposium on Principles of Programming Languages (POPL '15). 153-165.

[9] S. Chong, K. Vikram, and A. Myers. 2007. SIF: Enforcing Confidentiality and Integrity in Web Applications. In Proceedings of 16th USENIX Security Symposium. Article 1, 16 pages.

[10] B. Corcoran, N. Swamy, and M. Hicks. 2009. Cross-tier, Label-based Security Enforcement for Web Applications. In Proceedings of the 2009 ACM SIGMOD International Conference on Management of Data (SIGMOD '09). 269-282.

[11] C. Cotrini, T. Weghorn, and D. Basin. 2018. Mining ABAC Rules from Sparse Logs. In 2018 IEEE European Symposium on Security and Privacy (EuroS P). 31-46.

[12] M. Dalton, C. Kozyrakis, and N. Zeldovich. 2009. Nemesis: Preventing Authentication \& Access Control Vulnerabilities in Web Applications. In Proceedings of the 18th Conference on USENIX Security Symposium. 267-282.

[13] Data Policy 2019. https://www.facebook.com/policy.php.

[14] Django REST Framework 2017. https://www.django-rest-framework.org.

[15] Django: The web framework for perfectionists with deadlines 2019. https://www. djangoproject.com.

[16] M. E. Kabir, H. Wang, and E. Bertino. 2011. A Conditional Purpose-based Access Control Model with Dynamic Roles. Expert Syst. Appl. 38, 3 (2011), 1482-1489.

[17] Gramm-Leach-Bliley Act 2019. https://www.ftc.gov/enforcement/rules/ rulemaking-regulatory-reform-proceedings/financial-privacy-rule.

[18] D. B. Giffin, A. Levy, D. Stefan, D. Terei, D. Mazières, J. C. Mitchell, and A Russo. 2012. Hails: Protecting Data Privacy in Untrusted Web Applications. In Presented as part of the 10th USENIX Symposium on Operating Systems Design and Implementation (OSDI 12). USENIX, Hollywood, CA, 47-60.

[19] Google Privacy Policy 2019. https://policies.google.com/privacy.

[20] J. De Groot. 2019. The History of Data Breaches. https://digitalguardian.com/ blog/history-data-breaches.

[21] M. Guarnieri, M. Balliu, D. Schoepe, D. Basin, and A. Sabelfeld. 2019. InformationFlow Control for Database-Backed Applications. In Proceedings of the 4th IEEE European Symposium on Security and Privacy (EuroS\&P 2019). 79-94.

[22] Jiawei Han, Jian Pei, and Yiwen Yin. 2000. Mining Frequent Patterns without Candidate Generation. SIGMOD Rec. 29, 2 (May 2000), 1-12.

[23] Patricia Huey. 2014. Oracle Database Security Guide 11g Release 1 (11.1). https: //docs.oracle.com/cd/B28359_01/network.111/b28531/title.htm.

[24] Padmavathi Iyer and Amirreza Masoumzadeh. 2018. Mining Positive and Negative Attribute-Based Access Control Policy Rules. In Proceedings of the 23nd ACM on Symposium on Access Control Models and Technologies (SACMAT '18). Association for Computing Machinery, New York, NY, USA, 161-172.

[25] Jiang JX and Bai G. 2019. Evaluation of Causes of Protected Health Information Breaches. FAMA Intern Med. 179, 2 (2019), 265-267.

[26] K. LeFevre, R. Agrawal, V. Ercegovac, R. Ramakrishnan, Y. Xu, and D. DeWitt 2004. Limiting Disclosure in Hippocratic Databases. In Proceedings of the Thirtieth International Conference on Very Large Data Bases - Volume 30 (VLDB '04). VLDB Endowment, 108-119.

[27] A. Mehta, E. Elnikety, K. Harvey, D. Garg, and P. Druschel. 2017. Qapla: Policy compliance for database-backed systems. In 26th USENIX Security Symposium (USENIX Security 17). Vancouver, BC, 1463-1479.

[28] Microsoft Privacy Statement 2019. https://privacy.microsoft.com/en-us/ privacystatement.
[29] Decebal Mocanu, Fatih Turkmen, and Antonio Liotta. 2015. Towards ABAC Policy Mining from Logs with Deep Learning. In In proc. of the 18th International Multiconference, IS 2015, Intelligent Systems.

[30] D. Muthukumaran, D. O'Keeffe, C. Priebe, D. Eyers, B. Shand, and P. Pietzuch. 2015. FlowWatcher: Defending Against Data Disclosure Vulnerabilities in Web Applications. In Proceedings of the 22Nd ACM SIGSAC Conference on Computer and Communications Security (CCS '15). 603-615.

[31] A. Myers. 1999. JFlow: Practical Mostly-static Information Flow Control. In Proceedings of the 26th ACM SIGPLAN-SIGACT Symposium on Principles of Programming Languages (POPL '99). 228-241.

[32] Office for Civil Rights (OCR). 2013. The HIPAA Privacy Rule. https://www.hhs. gov/hipaa/for-professionals/privacy/index.html.

[33] J. Parker, N. Vazou, and M. Hicks. 2019. LWeb: Information Flow Security for Multi-tier Web Applications. Proc. ACM Program. Lang. 3, POPL, Article 75 (Jan. 2019), 75:1-75:30 pages.

[34] B. Parno, J. McCune, D. Wendlandt, D. Andersen, and A. Perrig. 2009. CLAMP: Practical Prevention of Large-Scale Data Leaks. In Proceedings of the 2009 30th IEEE Symposium on Security and Privacy (SP '09). 154-169.

[35] S. Rizvi, A. Mendelzon, S. Sudarshan, and P. Roy. 2004. Extending Query Rewriting Techniques for Fine-grained Access Control. In Proceedings of the 2004 ACM SIGMOD International Conference on Management of Data (SIGMOD). 551-562.

[36] B. Roberts. 2017. Open source social network built with Django and Django REST framework . http://vataxia.net/.

[37] Matthew W Sanders and Chuan Yue. 2019. Mining Least Privilege Attribute Based Access Control Policies. In Proceedings of the 35th Annual Computer Security Applications Conference (ACSAC '19). Association for Computing Machinery, New York, NY, USA, 404-416.

[38] D. Schoepe, D. Hedin, and A. Sabelfeld. 2014. SeLINQ: Tracking Information Across Application-database Boundaries. In Proceedings of the 19th ACM SIGPLAN International Conference on Functional Programming (ICFP '14). 25-38.

[39] D. Schultz and B. Liskov. 2013. IFDB: Decentralized Information Flow Control for Databases. In Proceedings of the 8th ACM European Conference on Computer Systems (EuroSys '13). 43-56.

[40] Securing DB2 2014. https://www.ibm.com/support/knowledgecenter/en/ SSEPEK_10.0.0/nav/src/tpc/db2z_securityintro.html.

[41] Security Enhanced PostgreSQL 2009. https://code.google.com/archive/p/ sepgsql/.

[42] S. Sen, S. Guha, A. Datta, S. Rajamani, J. Tsai, and J. Wing. 2014. Bootstrapping Privacy Compliance in Big Data Systems. In Proceedings of the 2014 IEEE Symposium on Security and Privacy (SP '14). 327-342.

[43] Spirit - Django based forum software 2019. https://spirit-project.com/.

[44] M. Stonebraker and E. Wong. 1974. Access Control in a Relational Data Base Management System by Query Modification. In Proceedings of the 1974 Annual Conference - Volume 1 (ACM '74). 180-186.

[45] European Union. 2016. Regulation (EU) 2016/679 of the European Parliament and of the Council of 27 April 2016 on the protection of natural persons with regard to the processing of personal data and on the free movement of such data. Official fournal of the European Union L119 (4 May 2016), 1-88.

[46] P. Upadhyaya, M. Balazinska, and D. Suciu. 2015. Automatic Enforcement of Data Use Policies with DataLawyer. In Proceedings of the 2015 ACM SIGMOD International Conference on Management of Data (SIGMOD '15). 213-225.

[47] Q. Wang, T. Yu, N. Li, J. Lobo, E. Bertino, K. Irwin, and J. Byun. 2007. On the Correctness Criteria of Fine-grained Access Control in Relational Databases. In Proceedings of the 33rd International Conference on Very Large Data Bases (VLDB '07). VLDB Endowment, 555-566.

[48] Zhongyuan Xu and Scott D. Stoller. 2012. Algorithms for Mining Meaningful Roles. In Proceedings of the 17th ACM Symposium on Access Control Models and Technologies (SACMAT). ACM Press, 57-66.

[49] Zhongyuan Xu and Scott D. Stoller. 2013. Mining Parameterized Role-Based Policies. In Proceedings of the Third ACM Conference on Data and Application Security and Privacy (CODASPY '13). Association for Computing Machinery, New York, NY, USA, 255-266.

[50] Zhongyuan Xu and Scott D. Stoller. 2014. Mining Attribute-Based Access Control Policies from Logs. In Proceedings of the 28th Annual IFIP WG 11.3 Working Conference on Data and Applications Security and Privacy XXVIII - Volume 8566 (DBSec 2014). Springer-Verlag, Berlin, Heidelberg, 276-291.

[51] Z. Xu and S. D. Stoller. 2015. Mining Attribute-Based Access Control Policies. IEEE Transactions on Dependable and Secure Computing 12, 5 (Sep. 2015), 533-545.

[52] J. Yang, T. Hance, T. Austin, A. Solar-Lezama, C. Flanagan, and S. Chong. 2016. Precise, Dynamic Information Flow for Database-backed Applications. In Proceedings of the 37th ACM SIGPLAN Conference on Programming Language Design and Implementation (PLDI '16). 631-647.

[53] J. Yang, K. Yessenov, and A. Solar-Lezama. 2012. A Language for Automatically Enforcing Privacy Policies. In Proceedings of the 39th Annual ACM SIGPLANSIGACT Symposium on Principles of Programming Languages (POPL '12). 85-96.

[54] A. Yip, X. Wang, N. Zeldovich, and M. F. Kaashoek. 2009. Improving Application Security with Data Flow Assertions. In Proceedings of the ACM SIGOPS 22nd Symposium on Operating Systems Principles (SOSP '09). 291-304. 